\title{
Tempered Generalized Functions $\zeta$ ' As an Ordered Topological Vector Space and Fourier Transform Of the Elements of $\zeta$ '
}

\author{
Geetha K.V. ${ }^{1}$, Mangalambal N.R. ${ }^{2}$ \\ ${ }^{I}$ Department of General Science, Sahrdaya College of Engineering and Technology, Kodakara, Thrissur Dist., \\ Kerala, India-680684 \\ ${ }^{2}$ Department of Mathematics, St. Joseph's College, Irinjalakuda, Kerala-680121
}

\begin{abstract}
The notion of 'order' is introduced on the linear space $\zeta$ ' of tempered generalized functions and the topology of bounded convergence is assigned to $\zeta$ '. It is proved that the order topology and the topology of bounded convergence on $\zeta$ ' are the same. That the operations of convolution and direct product of comparable elements in $\zeta$ ' are compatible with the lattice operations in $\zeta$ ' is also proved. Fourier Transform is applied to the elements of ' $\zeta^{\prime}$ and the properties of the transform and its inverse with the topology of bounded convergence assigned to $\zeta$ ' are studied. Illustrations of application of Fourier Transform to obtain fundamental solutions of non-zero linear differential equations with constant coefficients are given. Comparison of various solutions is also done.
\end{abstract}

Key Words: Fourier Transform, tempered generalized function, positive cone.

MSC (2010): 42A38, 46F12,46F05

\section{Introduction}

The space of $\zeta$ test (rapidly decreasing) functions and its dual $\zeta^{\prime}$, the tempered generalized functions have been studied by many authors. We have introduced the notion of order to test function spaces and their duals [1].Also the topology of bounded convergence is assigned to the dual spaces. In this paper we apply these ideas to $\zeta$ and its dual $\zeta$ '.

In Section 2 we consider $\zeta$ the topological vector space of test functions that decrease rapidly. An order is defined on $\zeta$ by identifying a positive cone in $\zeta$. The topology of bounded convergence is assigned to the dual $\zeta^{\prime}$, the linear space of tempered generalized functions. An order is defined to the dual $\zeta$ ' and we have assured that the order topology and the topology of bounded convergence on $\zeta^{\prime}$ are the same.

The direct product and convolution of the elements in $\zeta^{\prime}$ and their product with respect to the topology of bounded convergence on $\zeta^{\prime}$ are studied in Section 3. In this section we also prove that the direct product and the convolution of comparable elements in $\zeta^{\prime}$ are compatible with the lattice operations.

The Fourier Transform and its inverse applied to the elements of ' $\zeta$ ' and their properties with respect to the topology of bounded convergence are studied in Section 4.

In Section 5 the fundamental solutions in $\zeta$ ' of differential equations with constant coefficients are studied. Illustrations of the above idea and comparison of various solutions are also done in Section.5.

\section{The Space $\zeta$ Of Test Functions}

Let $\zeta$ denote the linear space of all complex valued infinitely differentiable functions defined on $\mathbf{R}^{\mathrm{n}}$ such that the functions and all their derivatives decrease faster than any power of $|\mathrm{x}|^{-1}$ as $|\mathrm{x}| \rightarrow \infty$

Define $\|\varphi\| \mathrm{p}=\sup _{\alpha \leq p}\left(1+|x|^{2}\right)^{p / 2}\left|D^{\alpha} \phi(x)\right|, \mathrm{x} \varepsilon \mathbf{R}^{\mathrm{n}}, \varphi \varepsilon \zeta, \mathrm{p}=0,1,2, \ldots \ldots \ldots$

where $D^{\alpha}=\frac{\partial^{\alpha}}{\partial_{x_{1}}^{\alpha_{1}} \partial_{x_{2}}^{\alpha_{2}} \ldots \ldots \partial_{x_{n}}^{\alpha_{n}}} \quad, \alpha=\alpha_{1}+\alpha_{2}+\ldots \ldots+\alpha_{n}, \alpha_{1}, \alpha_{2}, \ldots \ldots, \alpha_{n}$ are non-negative integers, $\left\{\|\|_{p}\right\}$ is a countable collection of seminorms and satisfy

$$
\|\varphi\|_{0} \leq\|\varphi\|_{1} \leq\|\varphi\|_{2} \leq \ldots \ldots ., \varphi \varepsilon \zeta .
$$

Convergence in $\zeta$ is defined as follows: Given a sequence $\left(\varphi_{\mathrm{k}}\right)$ of functions in $\zeta, \quad \varphi_{\mathrm{k}} \rightarrow 0$ as $\mathrm{k} \rightarrow \infty$ in $\zeta$ if for all $\mathrm{p}=0,1,2, \ldots \ldots .,\left\|\varphi_{\mathrm{k}}\right\|_{\mathrm{p}} \rightarrow 0$ as $\mathrm{k} \rightarrow \infty$. Equivalently, $\quad \varphi_{\mathrm{k}} \rightarrow 0$ as $\mathrm{k} \rightarrow \infty$ in $\zeta$ if $\mathrm{x}^{\beta} \mathrm{D}^{\alpha} \varphi(\mathrm{x}) \rightarrow 0$ uniformly for $\mathrm{x} \varepsilon \mathbf{R}^{\mathrm{n}}$ as $\mathrm{k} \rightarrow \infty$. Let $\zeta_{\mathrm{p}}$ denote the completion of $\zeta_{\text {in }}$ the $\mathrm{p}^{\text {th }}$ norm. Then $\zeta_{\mathrm{p}}$ is a Banach space. [ Let $\mathrm{J}: \mathrm{X} \rightarrow \mathrm{X}$ " be the canonical embedding of $X$ in $X$ " and let $X_{c}$ denote the closure of $J(X)$ in $X$ ". Then $X_{c}$ is a Banach space called the completion of X [2, p.131]]. Clearly

$\zeta_{\mathrm{p}+1} \mathrm{C}_{=} \zeta_{\mathrm{p},}, \mathrm{p}=0,1, \ldots \ldots$

and each embedding is continuous. Vladimirov [4] has proved that each embedding is totally continuous and $\zeta$ $=\bigcap_{\mathrm{p} \geq 0} \zeta_{\mathrm{p}}, \zeta$ is complete. 
By identifying a positive cone in $\zeta$, an order relation is defined on $\zeta$ as follows.

Definition 2.1. Restricting $\zeta$ to real-valued functions, an order relation is defined on $\zeta$ by identifying the positive cone to be the set of non-negative functions in $\zeta$. When the field of scalars is $\mathbf{C}$, the field of complex numbers, $\mathrm{C}+\mathrm{iC}$ is the positive cone in $\zeta$ which is also denoted as $\mathrm{C}$. $\mathrm{C}$ defines an order relation on $\zeta, \varphi \leq \psi$ if $\psi-\varphi \varepsilon \mathrm{C}$.

The ordered topological vector space of distributions. The test function space $\mathbf{D}(\mathrm{I})$, I any open set in $\mathbf{R}^{\mathrm{n}}$ consists of infinitely differentiable functions on I, having compact support convergence in $\mathbf{D}(\mathrm{I})$,is defined as follows: A sequence of functions $\left(\varphi_{k}\right)$ in $\mathbf{D}(\mathrm{I})$ converges to $\varphi$ in $\mathbf{D}(\mathrm{I})$ if there exists a bounded set I' such that $\overline{\mathrm{I}^{\prime}}$ $\mathrm{C}_{=} \mathrm{I}$, supp $\varphi_{\mathrm{k}} \mathrm{C}_{=} \mathrm{I}^{\prime}$ and for every $\alpha, \mathrm{D}^{\alpha} \varphi_{\mathrm{k}}(\mathrm{x}) \rightarrow \mathrm{D}^{\alpha} \varphi(\mathrm{x})$ uniformly on I as $\mathrm{k} \rightarrow \infty$. A linear set equipped with this convergence is called the space of test functions $\mathbf{D}(\mathrm{I})$. When $I=\mathbf{R}^{\mathrm{n}}$ we denote $\mathbf{D}\left(\mathrm{R}^{\mathrm{n}}\right)$ as $\mathbf{D}$.

By restricting $\mathbf{D}$ to real valued functions an order relation is defined on $\mathbf{D}$ by identifying the positive cone $\mathbf{C}$ to be the set of all non-negative functions of $\mathbf{D}$. When the field of scalars is $\mathbf{C}, \mathbf{C}+\mathrm{iC}$ is the positive cone of $\mathbf{D}$.

Theorem 2.1 $\mathbf{D}$ is a dense proper subset of $\zeta$, the order on $\mathbf{D}$ being the same as the order induced on $\mathbf{D}$ by $\zeta$.

Proof. For $\varphi \varepsilon \zeta$ the sequence of functions $\left(\varphi_{\mathrm{k}}\right)$ defined by

$$
\Phi_{\mathrm{k}}(\mathrm{x})=\varphi(\mathrm{x}) \eta(\mathrm{x} / \mathrm{k}), \mathrm{k}=1,2,3, \ldots \ldots \ldots
$$

where $\eta(x)=1,|x|<1, \eta \varepsilon \mathbf{D}$ is such that $\varphi_{\mathrm{k}} \rightarrow \varphi$ so that $\mathbf{D}$ is dense in $\zeta$. However $\exp \left(-|\mathrm{x}|^{2}\right) \varepsilon \zeta$ but not to $\mathbf{D}$. Since $\mathbf{D}$ is a linear subspace of $\zeta$, the cone in $\mathrm{D}$ induced by $\zeta$ is $\mathrm{C}_{\zeta} \cap \mathbf{D}$ where $\mathrm{C}_{\zeta}$ is the cone in $\zeta$. Clearly $\mathrm{C}_{\zeta} \cap$ $\mathbf{D}$ is the positive cone $\mathbf{C}_{\mathbf{D}}$ in $\mathbf{D}$. Hence the theorem.

Note 1. If $A$ is a subset of a vector space $E$ ordered by a cone $C$ the full hull $[A]$ of $A$ is defined by

$$
[\mathrm{A}]=\{\mathrm{z} \& \mathrm{E}: \mathrm{x} \leq \mathrm{z} \leq \mathrm{y} \text { for } \mathrm{x}, \mathrm{y} \& \mathrm{~A}\}
$$

i.e., $[\mathrm{A}]=(\mathrm{A}+\mathrm{C}) \cap(\mathrm{A}-\mathrm{C})$.

If $[\mathrm{A}]=\mathrm{A}$, then $\mathrm{A}$ is said to be a full set.

Note 2. If $\mathrm{E}(\tau)$ is an ordered topological vector space ordered by a positive cone then the cone $\mathrm{C}$ is said to be a normal cone for the topology $\tau$ if there exists a neighbourhood basis of 0 for $\tau$ consisting of full sets.

Note 3.

(a) A family $\mathbf{G} \neq\{\varphi\}$ of bounded subsets of a locally convex space $E$ is said to be saturated if (i) it contains arbitrary subsets of each of its members (ii) it contains all scalar multiples of each of its members(iii) it contains the closed convex circled hull of the union of each its finite subfamily.

(b) A fundamental system of bounded sets of a topological vector space $\mathrm{E}$ is a family $\mathbf{B}$ of bounded sets such that every bounded subset of $E$ is contained in a suitable member of $\mathbf{B}$.

(c) Suppose that $\mathrm{G}$ is a saturated class of $\tau$-bounded subsets of an ordered locally convex space $\mathrm{E}(\tau)$ such that $\mathrm{E}=\mathrm{U}\{\mathrm{S}$ : $\mathrm{S} \varepsilon \mathbf{G}\}$. The cone $\mathrm{C}$ in $\mathrm{E}(\tau)$ is a $\mathbf{G}$-cone (resp. strict $\mathbf{G}$-cone ) if the class

$$
\text { cl. } \mathrm{G}_{\mathrm{C}}=\{\text { cl. }(\mathrm{S} \cap \mathrm{C})-(\mathrm{S} \cap \mathrm{C}): \mathrm{S} \& \mathbf{G}\}
$$

(resp. $\left.\mathrm{G}_{\mathrm{C}}=\{(\mathrm{S} \cap \mathrm{C})-(\mathrm{S} \cap \mathrm{C}): \mathrm{S} \varepsilon \mathbf{G}\}\right)$ is a fundamental system for G. A G-cone (resp. strict G-cone) for the class $\mathrm{G}$ of all $\tau$-bounded sets in $\mathrm{E}(\tau)$ is called a b-cone (resp. strict b-cone).

The dual ' D' of $\mathbf{D}$ is called the space of distributions. D' is ordered by the dual cone C' of the cone $\mathbf{C}$ in $\mathbf{D}$. Peressini[3] has observed that the cone of $\mathbf{D}$ is a strict b-cone ( Example 1.17(c), Chapter 2, D considered as a real vector space) and that the dual cone $C^{\prime}$ in $\mathbf{D}^{\prime}$ is normal with respect to the topology of bounded convergence on D'.

Theorem 2.2 The cone of $\zeta$ is not normal.

Proof. We have observed that $\mathbf{D}$ is a subspace of $\zeta$ and that $\mathbf{C}_{\zeta} \cap \mathbf{D}=\mathbf{C}_{\mathbf{D}}$. Peressini[3] has observed that if $\mathrm{K}$ is a normal cone in an ordered topological vector space $E(\tau)$ and if $M$ is a linear subspace of $E$ then $K \cap M$ is a normal cone in $\mathrm{M}$ for the subspace topology. Applying this result, using Theorem 2.1, it follows that the cone of $\zeta$ is not normal. $\square$

Theorem 2.3. The cone $\mathrm{C}$ is a strict b-cone in $\zeta$.

Proof. Let $\zeta$ be restricted to real-valued functions. Let $\mathbf{B}$ be the saturated class of all bounded, circled subsets of $\zeta$ for $\tau$. Then $\zeta=\mathrm{U}_{\mathrm{B} \varepsilon \mathbf{B}}$ where $\mathbf{B}_{\mathrm{C}}=\{(\mathrm{B} \cap \mathrm{C})-(\mathrm{B} \cap \mathrm{C})$ : $\mathrm{B} \varepsilon \mathbf{B}\}$ is a fundamental system for $\zeta$ and $\mathrm{C}$ is a strict bcone, since $\mathbf{B}$ is the collection of all $\tau$-bounded sets in $\zeta$.

Let B be a bounded circled subset of $\zeta$ for $\tau$. Then all functions in B have their support in some compact set $\mathrm{K}_{\mathrm{j} 0}$ and there exists a constant $\mathrm{M}>0$ such that $\quad|\varphi(\mathrm{t})| \leq \mathrm{M}$, for all $\varphi \varepsilon \mathrm{B}, \mathrm{t} \varepsilon \mathrm{K}_{\mathrm{j} 0}$.

Let $\psi(\mathrm{t})=\mathrm{M}, \mathrm{t} \varepsilon \mathrm{K}_{\mathrm{j} 0+1}$

$$
=0, \mathrm{t} \text { not } \varepsilon \mathrm{K}_{\mathrm{j} 0+1} \text {. }
$$

Then the regularization $\xi$ of $\psi$ is defined by

$\xi(\mathrm{t})=\int_{\mathbf{R n}} \theta_{\alpha}\left(\mathrm{t}-\mathrm{t}^{\prime}\right) \psi\left(\mathrm{t}^{\prime}\right) \mathrm{dt}$ ' and $\xi$ has its support in $\mathrm{K}_{\mathrm{j} 0+2 \text {. }}$

Also, $\mathrm{B} \mathrm{C}_{=}(\mathrm{B}+\xi) \cap\{\xi\}$

$\mathrm{C}_{=}(\mathrm{B}+\xi) \cap \mathrm{C}-(\mathrm{B}+\xi) \cap \mathrm{C}$.

It follows that $\mathrm{C}$ is a strict $\mathrm{b}$-cone. We conclude that $\mathrm{C}+\mathrm{iC}$ is a strict $\mathrm{b}$-cone. $\square$ 


\section{Direct Product And Convolution Of Tempered Generalized Functions}

Definition 3.1. Let $\mathrm{f}(\mathrm{x})$ and $\mathrm{g}(\mathrm{y})$ be locally integrable functions in open sets $\quad \mathrm{I}_{1} \mathrm{C}_{=} \mathbf{R}^{\mathrm{n}}$ and $\mathrm{I}_{2} \mathrm{C}_{=} \mathbf{R}^{\mathrm{m}}$ respectively. The function $f(x) g(y)$ is locally integrable in $I_{1} \times I_{2}$ and defines a generalized function $f(x) g(y)=$ $\mathrm{g}(\mathrm{y}) \mathrm{f}(\mathrm{x})$ in $\mathbf{D}^{\prime}\left(\mathrm{I}_{1} \times \mathrm{I}_{2}\right)$ operating on test functions $\varphi(\mathrm{x}, \mathrm{y})$ in $\mathbf{D}\left(\mathrm{I}_{1} \times \mathrm{I}_{2}\right)$ via the formula

$<\mathrm{f}(\mathrm{x}) \mathrm{g}(\mathrm{y}), \varphi>=\int_{\mathrm{I} 1 \times \mathrm{II}} \mathrm{f}(\mathrm{x}) \mathrm{g}(\mathrm{y}) \varphi(\mathrm{x}, \mathrm{y}) \mathrm{dx} d \mathrm{y}$

$$
\begin{aligned}
& =\int_{I 1} \mathrm{f}(\mathrm{x}) \int_{\mathrm{I} 2} \mathrm{~g}(\mathrm{y}) \varphi(\mathrm{x}, \mathrm{y}) d \mathrm{xdy} \\
& =\int_{\mathrm{I1}} \mathrm{x} \mathrm{I2} \mathrm{g}(\mathrm{y}) \mathrm{f}(\mathrm{x}) \varphi(\mathrm{x}, \mathrm{y}) d \mathrm{x} d \mathrm{y}
\end{aligned}
$$$$
=\int_{12} \mathrm{~g}(\mathrm{y}) \int_{\mathrm{I} 1} \mathrm{f}(\mathrm{x}) \varphi(\mathrm{x}, \mathrm{y}) d \mathrm{x} d \mathrm{y}
$$

i.e., $\langle\mathrm{f}(\mathrm{x}) \mathrm{g}(\mathrm{y}), \varphi>=<\mathrm{f}(\mathrm{x})<\mathrm{g}(\mathrm{y}), \varphi(\mathrm{x}, \mathrm{y})>>$

$<\mathrm{g}(\mathrm{y}) \mathrm{f}(\mathrm{x}), \varphi>=\langle\mathrm{g}(\mathrm{y})<\mathrm{f}(\mathrm{x}), \varphi(\mathrm{x}, \mathrm{y})>>$.

Definition 3.2. For $\mathrm{f}(\mathrm{x}) \varepsilon \zeta^{\prime}\left(\mathbf{R}^{\mathrm{n}}\right), \mathrm{g}(\mathrm{y}) \varepsilon \zeta^{\prime}\left(\mathbf{R}^{\mathrm{m}}\right)$, since $\zeta^{\prime} \mathrm{C}_{=} \mathbf{D}^{\prime}$ the direct product $\mathrm{f}(\mathrm{x}) \mathrm{x} \mathrm{g}(\mathrm{y})$ is a generalized function in $\mathbf{D}^{\prime}\left(\mathbf{R}^{\mathrm{n}+\mathrm{m}}\right)$ defined by

$$
<\mathrm{f}(\mathrm{x}) \mathrm{x} g(\mathrm{y}), \varphi>=<\mathrm{f}(\mathrm{x})<\mathrm{g}(\mathrm{y}), \varphi(\mathrm{x}, \mathrm{y})>>\text {. }
$$

Note. If $\mathrm{f}(\mathrm{x}) \varepsilon \zeta^{\prime}\left(\mathbf{R}^{\mathrm{n}}\right), \mathrm{g}(\mathrm{y}) \varepsilon \zeta^{\prime}\left(\mathbf{R}^{\mathrm{m}}\right), \mathrm{f}(\mathrm{x}) \mathrm{x} \mathrm{g}(\mathrm{y}) \varepsilon \zeta^{\prime}\left(\mathbf{R}^{\mathrm{n}+\mathrm{m}}\right)$.

Theorem 3.1. The direct product is an order preserving continuous operation from $\zeta^{\prime}\left(\mathbf{R}^{\mathrm{n}}\right)$ to $\zeta^{\prime}\left(\mathbf{R}^{\mathrm{n}+\mathrm{m}}\right)$ when $\zeta^{\prime}$ is assigned the topology of bounded convergence.

Proof. The fact that the direct product is a continuous operation from $\zeta^{\prime}\left(\mathbf{R}^{\mathrm{n}}\right)$ to $\quad \zeta^{\prime}\left(\mathbf{R}^{\mathrm{n}+\mathrm{m}}\right)$ when $\zeta^{\prime}$ is assigned the topology of bounded convergence follows from Theorem2.15,[1].

Let $\varphi(\mathrm{x}, \mathrm{y}) \geq 0, \mathrm{f} \geq 0, \mathrm{~g} \geq 0$.

Since $<\mathrm{f}(\mathrm{x}) \mathrm{x} \mathrm{g}(\mathrm{y}), \varphi(\mathrm{x}, \mathrm{y})>=<\mathrm{f}(\mathrm{x})<\mathrm{g}(\mathrm{y}), \varphi(\mathrm{x}, \mathrm{y})>>$ it follows that $\mathrm{f} \mathrm{x} \mathrm{g} \geq 0$.

Definition 3.3. Let $f$ and $g$ be locally integrable functions defined on $\mathbf{R}^{\mathrm{n}}$. If

$\int \mathrm{f}(\mathrm{y}) \mathrm{g}(\mathrm{x}-\mathrm{y}) \mathrm{dy}$ exists for almost all $x \varepsilon \mathbf{R}^{\mathrm{n}}$ and defines a locally integrable function on $\mathbf{R}^{\mathrm{n}}$ and the convolution of $\mathrm{f}$ and $\mathrm{g}$ denoted as $\mathrm{f}$ $* \mathrm{~g}$ is defined by

Note 1. It can be proved that

$$
\begin{aligned}
\left(f^{*} g\right)(x) & =\int f(y) g(x-y) d y \\
& =\int g(y) f(x-y) d y \\
& =(g * f)(x)
\end{aligned}
$$

$\int f(x) g(y) \varphi(x+y) d x d y=\lim _{k \rightarrow \infty} \int f(x) g(y) \eta_{k}(x ; y) \varphi(x+y) d x d y$

where $\eta_{\mathrm{k}}$ is any sequence of functions taken from $\zeta\left(\mathbf{R}^{2 \mathrm{n}}\right)$ that converges to 1 in $\mathbf{R}^{2 \mathrm{n}}$.

Note 2. For $\mathrm{f}, \mathrm{g} \varepsilon \zeta^{\prime}\left(\mathbf{R}^{\mathbf{n}}\right)$ the convolution $\mathrm{f} * \mathrm{~g}$ is the functional

$<\mathrm{f} * \mathrm{~g}, \varphi>=\lim _{\mathrm{k} \rightarrow \infty}<\mathrm{f}(\mathrm{x}) \mathrm{x} \mathrm{g}(\mathrm{y}), \eta_{\mathrm{k}}(\mathrm{x} ; \mathrm{y}) \varphi(\mathrm{x}+\mathrm{y})>$,

$\Phi \varepsilon \zeta\left(\mathbf{R}^{\mathbf{n}}\right)$ and $\eta_{\mathrm{k}}$ is any sequence of functions taken from $\zeta\left(\mathbf{R}^{2 \mathrm{n}}\right)$ that converges to 1 in $\mathbf{R}^{2 \mathrm{n}}$. The limit is independent of $\left(\eta_{\mathrm{k}}\right)$.

Definition 3.4. Let $f, g \varepsilon \zeta^{\prime}$. Then

$\mathrm{f} * \mathrm{~g}=\lim _{\mathrm{k} \rightarrow \infty} \mathrm{f} \eta_{\mathrm{k}}{ }^{*} \mathrm{~g}$ in $\zeta^{\prime}$, if the above limit exists for any $\left(\eta_{\mathrm{k}}\right)$ converging to 1 in $\mathbf{R}^{\mathrm{n}}$.

Note. If the above limit exists it does not depend on $\eta \mathrm{k}$ and there exists a convolution $\mathrm{f} * \mathrm{~g}$.

Also $\mathrm{f} * \mathrm{~g}=\mathrm{g} * \mathrm{f}$

Theorem 3.2. Let $\mathrm{f} \varepsilon \zeta^{\prime}, \mathrm{g} \varepsilon \mathbf{E}^{\prime}$. Then the convolution $\mathrm{f}^{*} \mathrm{~g} \varepsilon \zeta^{\prime}$ and can be represented as

$<\mathrm{f} * \mathrm{~g}>=<\mathrm{f}(\mathrm{x}) \mathrm{Xg}(\mathrm{x}), \eta(\mathrm{y}) \Phi(\mathrm{x}+\mathrm{y})>, \Phi \varepsilon \zeta$,

where $\eta$ is any function in $\mathbf{D}$ equal to 1 in a neighbourhood of the support of $g$. The operation $f \rightarrow f * g$ is order preserving and continuous from $\zeta^{\prime}$ and $\mathrm{g} \rightarrow \mathrm{f}{ }^{*} \mathrm{~g}$ is order preserving and continuous from $\mathbf{E}^{\prime}$ to $\zeta^{\prime}$ when the topology of bounded convergence is assigned to $\zeta$ ' and $\mathbf{E}$ '.

Proof. The continuity of $\mathrm{f} \rightarrow \mathrm{f} * \mathrm{~g}$ from $\zeta^{\prime}$ to $\zeta^{\prime}$ and $\mathrm{g} \rightarrow \mathrm{f} * \mathrm{~g}$ from $\mathbf{E}^{\prime}$ to $\zeta^{\prime}$ follow by Theorem $2.15,[1]$

Let $\mathrm{f} \geq 0, \mathrm{~g} \geq 0, \Phi \geq 0$. Since

$$
\begin{aligned}
& <\mathrm{f}(\mathrm{x}) \mathrm{Xg}(\mathrm{x}), \eta_{\mathrm{k}}(\mathrm{x} ; \mathrm{y}) \Phi(\mathrm{x}+\mathrm{y}) \geq 0 \\
& \text { for } \eta_{\mathrm{k}}(\mathrm{x})=\eta(\mathrm{x} / \mathrm{k}), \mathrm{k}=1,2,3, \ldots \ldots \ldots \ldots \\
& \\
& =1, \text { if }|\mathrm{x}|
\end{aligned}
$$

and since

$$
\lim _{\mathrm{k} \rightarrow \infty}<\mathrm{f}(\mathrm{x}) \mathrm{x} \mathrm{g}(\mathrm{x}), \eta_{\mathrm{k}}(\mathrm{x} ; \mathrm{y}) \Phi(\mathrm{x}+\mathrm{y})>
$$

if it exits is independent of $\left(\eta_{\mathrm{k}}\right)$, it follows $\mathrm{f}^{*} \mathrm{~g} \geq 0$. $\square$

Theorem 3.3 The operations of convolution and direct product of comparable elements in $\zeta$ ' are compatible with lattice operations i.e., if $\mathrm{f}_{1}, \mathrm{f}_{2}$ and $\mathrm{g}_{1}, \mathrm{~g}_{2}$ are comparable elements in $\zeta^{\prime}$ then $\mathrm{f}_{\mathrm{i}} * \mathrm{~g}_{\mathrm{i}}, \mathrm{i}=1,2$ and $\mathrm{f}_{\mathrm{i}} X \mathrm{~g}_{\mathrm{i}}, \mathrm{i}=1,2$ are also comparable and

$$
\begin{aligned}
& \left(f_{1} * g_{1}\right) \vee\left(f_{2} * g_{2}\right)=\left(f_{1} \vee f_{2}\right) *\left(g_{1} \vee g_{2}\right) \\
& \left(f_{1} * g_{1}\right) \wedge\left(f_{2} * g_{2}\right)=\left(f_{1} \wedge f_{2}\right) *\left(g_{1} \wedge g_{2}\right) \\
& \text { and } \\
& \left(f_{1} X g_{1}\right) \vee\left(f_{2} X g_{2}\right)=\left(f_{1} \vee f_{2}\right) X\left(g_{1} \vee g_{2}\right)
\end{aligned}
$$


$\left(f_{1} X g_{1}\right) \wedge\left(f_{2} X g_{2}\right)=\left(f_{1} \wedge f_{2}\right) X\left(g_{1} \wedge g_{2}\right)$

Proof. For $f \geq 0, g \geq 0$ we have

$\int \mathrm{f} \vee \mathrm{g}=\int \mathrm{f} \vee \int \mathrm{g}$ and $\quad \int \mathrm{f} \wedge \mathrm{g}=\int \mathrm{f} \wedge \int \mathrm{g}$

From the definition of convolution and direct product in ' $\zeta^{\prime}$ the theorem follows. $\square$

\section{Fourier Transform Of Tempered Generalized Functions}

Definition 4.1. If $\mathrm{f}$ is an integrable function on $\mathbf{R}^{\mathbf{n}}$ its Fourier transform is defined as

$F(f)(\xi)=\int_{R n} f(x) e^{i(\xi) x)} d x$

which is a continuous function on $\mathbf{R}^{\mathbf{n}}$ and hence determines a regular tempered generalized function. For $\varphi \varepsilon \zeta, f$ $\varepsilon \zeta^{\prime}$ the Fourier transform of $\mathrm{f}$ is defined as

$<\mathrm{F}(\mathrm{f}), \varphi>=<\mathrm{f}, \mathrm{F}(\varphi)>$.

Note 1. Since the operation $\varphi \rightarrow F(\varphi)$ is linear and continuous from $\zeta$ to $\zeta$ and since the topology of pointwise convergence is weaker than the topology of bounded convergence on $\zeta^{\prime}, F(f)$ is a member of $\zeta^{\prime}$ and the operation $\mathrm{f} \rightarrow \mathrm{F}(\mathrm{f})$ is linear and continuous on $\zeta^{\prime}$ with the topology of bounded convergence assigned to $\zeta^{\prime}$.

Note 2. From the general theory of Fourier transformation the function $\varphi(x)$ is expressed in terms of its Fourier transform $\mathrm{F}(\varphi)(\xi)$ with the aid of the inverse Fourier transform $\mathrm{F}^{-1}$ as

$\varphi=\mathrm{F}^{-1}(\mathrm{~F}(\varphi))=\mathrm{F}\left(\mathrm{F}^{-1}(\varphi)\right)$.

$\mathrm{F}^{-1}(\psi)(\mathrm{x})=\frac{1}{(2 \pi)^{n}} \int_{R^{n}} \psi(\xi) e^{-i(x \cdot \xi)} d \xi$

$=\frac{1}{(2 \pi)^{n}} F(\psi)(-x)$

$$
=\frac{1}{(2 \pi)^{n}} \int_{R^{n}} \psi(-\xi) e^{i(x \cdot \xi)} d \xi
$$

$=\frac{1}{(2 \pi)^{n}} F(\psi)(-\xi)$

Definition 4.2. For $\mathrm{f} \varepsilon \mathrm{C}^{\prime} \cap \zeta^{\prime}, \mathrm{F}^{-1}(\mathrm{f})$ is defined as

$\mathrm{F}^{-1}(\mathrm{f})=\frac{1}{(2 \pi)^{n}} F(f(-x))$.

Theorem 4.1. The operation $\mathrm{f} \rightarrow \mathrm{F}$ (f) is an isomorphism $\mathrm{C}^{\prime} \cap \zeta^{\prime}$ and hence on $\zeta^{\prime}$ when $\zeta^{\prime}$ is assigned the topology of bounded convergence.

Definition 4.3. Let $\mathrm{f}(\mathrm{x}, \mathrm{y}) \varepsilon\left(\zeta, \mathbf{R}^{\mathrm{n}+\mathrm{m}}\right)$ where $\mathrm{x} \varepsilon \mathbf{R}^{\mathbf{n}}, \mathrm{y} \varepsilon \mathbf{R}^{\mathrm{m}}$. The restricted Fourier transform $\mathrm{F}_{\mathrm{x}}$ (f) with respect to $\mathrm{x}=\left(\mathrm{x}_{1}, \mathrm{x}_{2}, \ldots \ldots, \mathrm{x}_{\mathrm{n}}\right)$ acts on $\varphi(\xi, \mathrm{y}) \varepsilon \varsigma\left(\mathbf{R}^{\mathbf{n}+\mathbf{m}}\right)$ by the equation

$$
<\mathrm{F}_{\mathrm{x}}(\mathrm{f}), \varphi>=<\mathrm{f}, \mathrm{F}_{\xi}(\varphi)>\text {. }
$$

Theorem 4.2. The operation $f \rightarrow F_{x}$ (f) is an isomorphism of $\zeta^{\prime}\left(\mathbf{R}^{\mathbf{n}+\mathbf{m}}\right)$ onto itself when the topology of bounded convergence is assigned to $\zeta^{\prime}\left(\mathbf{R}^{\mathbf{n}+\mathbf{m}}\right)$.

Proof. The operation $\varphi(\xi, \mathrm{y}) \rightarrow \mathrm{F}_{\xi}(\varphi)(\mathrm{x}, \mathrm{y})=\int_{R^{n}} \phi(x, y) e^{i(x \cdot \xi)} d x$ is a linear isomorphism of $\mathrm{C} \cap \zeta\left(\mathbf{R}^{\mathbf{n}+\mathbf{m}}\right)$ onto $\mathrm{C} \cap \zeta\left(\mathbf{R}^{\mathbf{n}+\mathbf{m}}\right)$ so that the above formula defines a generalized function $\mathrm{F}_{\mathrm{x}}(\mathrm{f})(\xi$, y $)$ in $\zeta^{\prime}\left(\mathbf{R}^{\mathbf{n}+\mathbf{m}}\right)$ when the topology of bounded convergence is assigned to $\zeta^{\prime}\left(\mathbf{R}^{\mathbf{n}+\mathbf{m}}\right)$.

The operation of inversion of the above restricted Fourier transform is defined by

$\mathrm{F}_{\xi}(\mathrm{g})=\frac{1}{(2 \pi)^{n}} F_{\xi}(g(-\xi, y))(x, y), \mathrm{g} \varepsilon \zeta^{\prime}\left(\mathbf{R}^{\mathbf{n}+\mathbf{m}}\right)$

which is also a continuous operation on $\zeta^{\prime}\left(\mathbf{R}^{\mathbf{n}+\mathbf{m}}\right)$ when the topology of bounded convergence is assigned to $\zeta^{\prime}$ $\left(\mathbf{R}^{\mathbf{n}+\mathbf{m}}\right)$. 


\section{Some Applications Of Fourier Transforms}

Fundamental solutions in D'. If $\mathrm{P}(\partial)=\sum_{|\alpha| \leq m} a_{\alpha} \partial^{\alpha}, \sum_{|\alpha|=m}\left|a_{\alpha}\right| \neq 0$ is a linear partial differential operator of the $\mathrm{m}^{\text {th }}$ order with constant coefficients , there exists $\varepsilon(\mathrm{x})$ in $\mathbf{D}^{\prime}$ ' such that
$\mathrm{P}(\partial) \varepsilon(\mathrm{x})=\delta(\mathrm{x})$
(1)

We call $\varepsilon(\mathrm{x})$ a fundamental solution of $\mathrm{P}(\partial)$ in $\mathbf{D}^{\prime}$. Every differential operator with constant coefficients $\mathrm{P}(\partial)$ $\neq 0$ has a fundamental solution in $\mathbf{D}^{\prime}[4]$.

Having a fundamental solution $\varepsilon$ of the operator $\mathrm{P}(\partial)$ we can construct a solution

$$
P(\partial) \mathrm{u}=\mathrm{f}, \mathrm{f} \varepsilon \mathbf{D} \text { ' }
$$

in the form of the convolution

$u \in D^{\prime}$ of the equation

$\mathrm{u}=\varepsilon * \mathrm{f}$

for those $f \varepsilon \mathbf{D}^{\prime}$ for which this convolution exists in D'. The solution of equation (2) is unique in the class of the generalized functions from D' for which the convolution with $\varepsilon$ exists.

\section{Some properties of the Fourier transform}

(1) Differentiating a Fourier transform

$\partial^{\alpha} \mathrm{F}(\mathrm{f})=\mathrm{F}\left((\mathrm{ix})^{\alpha} \mathrm{f}\right), \mathrm{f} \varepsilon \zeta^{\prime}$.

Putting $\mathrm{f}=1$ since $\mathrm{F}(1)=(2 \pi)^{\mathrm{n}} \delta(\xi)$

$\mathrm{F}\left(\mathrm{x}^{\alpha}\right)=(-1)^{|\alpha|} \partial^{\alpha} \mathrm{F}(1)=(2 \pi)^{\mathrm{n}}(-\mathrm{i})^{|\alpha|} \partial^{\alpha} \varsigma(\xi)$.

(2) The Fourier transform of a derivative.

$$
\begin{aligned}
F(f(x) X g(y) & =F_{x}(f(x) X F(g)(\eta)) \\
& =F y(F(f)(\xi) X g(y)) \\
& =F(f)(\xi) X F(g)(\eta) .
\end{aligned}
$$

Analogous formulas hold true also for the Fourier transform $\mathrm{F}_{\mathrm{x}}$. For example, $\partial_{\xi}^{\alpha} \partial_{\xi}^{\alpha} F_{x}(f)=F_{x}\left((i x)^{\alpha} \partial_{y}^{\beta}(f)\right)$

$F_{x}\left(\partial_{\xi}^{\alpha} \partial_{y_{y}}^{\beta} f\right)=(-i \xi)^{\alpha} \partial_{y}^{\beta} F_{x}(f)$

Fundamental solution in $\zeta$ '. The equation (1) in the class $\zeta^{\prime}$ is equivalent to the algebraic equation

(2) $\quad P(-i \xi) \varepsilon^{\sim}(\xi)=1$

with respect to the Fourier transform $\mathrm{F}(\varepsilon)=\varepsilon^{\sim}$.

Thus the problem of seeking a tempered fundamental solution turns out to be the problem of finding a solution $u$ in $\zeta$ ' of the equation $\mathrm{P}(\xi) \mathrm{u}=\mathrm{f}$

where $\mathrm{P} \neq 0$ is a polynomial and $\mathrm{f}$ is a specified generalized function in $\zeta$.

It has been proved that every non- zero linear differential equation with constant coefficients has a tempered fundamental solution.

Illustration. 1) A fundamental solution of the heat conduction equation $\frac{\partial \varepsilon}{\partial t}-a^{2} \Delta \varepsilon=\delta(x, t)$ where $\Delta \equiv$ $\partial^{2} / \partial \mathrm{x}^{2}$ is $\varepsilon(\mathrm{x}, \mathrm{t})=\frac{\theta(t)}{(2 a \sqrt{\pi t})^{n}} e^{\frac{-|x|^{2}}{4 a^{2} t}}$

Corresponding to $\mathrm{x}=\mathrm{x}_{1}, \mathrm{t}=\mathrm{t}_{1}, \mathrm{t}=\mathrm{t}_{2}$ we get two different solutions

$$
\begin{gathered}
\varepsilon\left(\mathrm{x}_{1}, \mathrm{t}_{1}\right)=\frac{\theta\left(t_{1}\right)}{(2 a \sqrt{\pi t})_{1}} e^{\frac{-\left|x_{1}\right|^{2}}{4 a^{2} t_{1}}} \\
\varepsilon\left(\mathrm{x}_{1}, \mathrm{t}_{2}\right)=\frac{\theta\left(t_{2}\right)}{\left(2 a \sqrt{\pi t}_{2}\right)^{n}} e^{\frac{-\left|x_{1}\right|^{2}}{4 a^{2} t_{2}}}
\end{gathered}
$$

The two solutions obtained corresponding to two different values of $t$, say $t=t_{1}$ and $t=t_{2}$ are comparable since $t_{1} \leq t_{2} \rightarrow \varepsilon\left(x_{1}, t_{1}\right) \geq \varepsilon\left(x_{1}, t_{2}\right)$. On the other hand, the two solutions obtained corresponding to two different values of $x$, say $x=x_{1}, x=x_{2}, t=t_{1}$ satisfy the relation

$\mathrm{x}_{1} \leq \mathrm{x}_{2} \rightarrow \varepsilon\left(\mathrm{x}_{1}, \mathrm{t}_{1}\right) \leq \varepsilon\left(\mathrm{x}_{2}, \mathrm{t}_{1}\right)$.

2. Fundamental solution of the Schrodinger operator 


$$
i \frac{\partial \varepsilon}{\partial t}+\frac{1}{2 m} \Delta \varepsilon=\delta(x, t)
$$

is

$$
\varepsilon(\mathrm{x}, \mathrm{t})=-\frac{1+i}{\sqrt{2}} \theta(t) \sqrt{\frac{m}{2 \pi t}} e^{i \frac{m}{2 t} x^{2}}
$$

For a fixed value of $\mathrm{x}$, say, $\mathrm{x}=\mathrm{x}_{1}$, the two solutions $\varepsilon\left(\mathrm{x}_{1}, \mathrm{t}_{1}\right)$ and $\varepsilon\left(\mathrm{x}_{1}, \mathrm{t}_{2}\right)$ are comparable since

$$
\mathrm{t}_{1} \leq \mathrm{t}_{2} \rightarrow \varepsilon\left(\mathrm{x}_{1}, \mathrm{t}_{1}\right) \geq \varepsilon\left(\mathrm{x}_{1}, \mathrm{t}_{2}\right) .
$$

Also for $\mathrm{t}=\mathrm{t}_{1}, \mathrm{x}=\mathrm{x}_{1}, \mathrm{x}=\mathrm{x}_{2}$ the solutions $\varepsilon\left(\mathrm{x}_{1}, \mathrm{t}_{1}\right)$ and $\varepsilon\left(\mathrm{x}_{2}, \mathrm{t}_{1}\right)$ satisfy

$$
\mathrm{x}_{1} \leq \mathrm{x}_{2} \rightarrow \varepsilon\left(\mathrm{x}_{1}, \mathrm{t}_{1}\right) \leq \varepsilon\left(\mathrm{x}_{2}, \mathrm{t}_{1}\right) \text {. }
$$

\section{Conclusion.}

The comparable solutions in $\zeta^{\prime}$ of a partial differential equation with constant coefficients form a chain in the partially ordered linear space with the topology of bounded convergence assigned to it. Hence the various lattice properties of this subclass may be subjected for further study.

\section{Acknowledgment.}

The authors gratefully acknowledge the guidance and support of Dr. T. Thrivikraman in the preparation of this paper.

\section{References}

[1] Geetha K.V. and Mangalambal N.R., On Dual Spaces of Ordered Multinormed Spaces and Countable Union Spaces, Bull. KMA, Vol.4, No.2 (Dec 2007) pp 63-75.

[2] Limaye B.V., Functional Analysis ( $2^{\text {nd }}$ Edition), New Age International Ltd., New Delhi, 1996

[3] Peressini, A.L., Ordered Topological Vector Spaces, Harper \& Row, New York, 1967.

[4] Vladimirov V.S., Methods of the Theory of Generalized Functions, Taylor and Francis, New York, 2002 